\title{
Vorbemerkung: Das Krisenmonster
}

In den Jahren von 2008 bis 2013 wird der mediale und öffentliche Diskurs massiv vom Eindruck einer global um sich greifenden Krise bestimmt. Beginnend mit dem Platzen der Immobilienblase in den USA wird zunächst ein gravierender Einbruch der Kurse auf den Finanzmärkten festgestellt. Große, systemrelevante Banken geraten weltweit ins Wanken und können nur durch immense staatliche Unterstützung vor dem Bankrott gerettet werden. ${ }^{1}$ Die Banken- und Finanzmarktkrise schlägt sich zusätzlich auf den Absatzmärkten nieder und bedingt wiederum eine weltweite Wirtschaftskrise. ${ }^{2}$ Es kommt zu einem rapiden Anwachsen der Staatsschulden, einigen europäischen Ländern droht sogar der Staatsbankrott. Die Zahlungsunfähigkeit dieser Euro-Staaten stellt nun eine erhebliche Gefährdung für die gesamte europäische Währungsunion dar. ${ }^{3}$ Die Krise ist somit über Jahre hinweg das die öffentliche Wahrnehmung bestimmende Thema. In der Gesellschaft breitet sich ein Gefühl der Bedrohung und der völligen Unsicherheit bezüglich der zukünftigen Entwicklung aus. Gerade die komplexen und schwer durchschaubaren Zusammenhänge eines Wirtschaftsund Finanzmarkts, der auf abstrakten Börsenspekulationen aufbaut und gleichzeitig die direkten Lebensumstände der Bevölkerung beeinflusst, verstärken das vorherrschende Gefühl des Kontrollverlustes. Die ausufernde Ereignislage wird dabei in der öffentlichen Diskussion und medialen Berichterstattung stets vereinfacht unter dem Begriff der ,Krise“ zusammengefasst. Es wird laufend über Treffen führender RegierungspolitikerInnen und ZentralbankvertreterInnen berichtet, die eine Katastrophe durch das Schnüren milliardenschwerer Rettungspakete in letzter Minute abwenden sollen. Nach und nach nehmen Ende 2013 und Anfang 2014 die Berichte zum Krisengeschehen der Wirtschaft und Staatsfinanzen ab und werden vom neuen Brennpunktthema der Flüchtlingsbewegung abgelöst. Die öffentliche Aufmerksamkeit wendet sich

1 Vgl. hierzu Dombret, A. \& Kenadjian, P. (Hg.) 2015. Too Big to Fail III: Structural Proposals. Should We Break Up the Banks? Berlin/ München: De Gruyter. Pflock, T. 2014. Europäische Bankenregulierung und das „Too big to fail-Dilemma“. Berlin: BWV. Sandberger, G. 2016. Die rechtliche Ordnung von Geld, Währung und Banken: Erfahrungen aus der Euro- und Bankenkrise. Marburg: Metropolis.

2 Vgl. hierzu Thiessen, N. 2016. Die Finanz- und Wirtschaftskrise von 2008 - Deutschland, Frankreich, Vereinigtes Königreich: eine Analyse von Krisenverläufen und -reaktionen vor dem Hintergrund vergleichender Kapitalismusforschung. Stuttgart: Franz Steiner.

3 Vgl. hierzu Brunnermeier, M., James, H. \& Landau, J.-P. 2016. The Euro and the Battle of Ideas. Oxford: Princeton University. Krieger, T. (Hg.) 2016. Europe's Crisis: the conflict theoretical perspective. Baden-Baden: Nomos. 
anderen Krisen zu, ohne dass ein abschließendes Verständnis oder eine Aufarbeitung des Geschehens vollzogen wurde. ${ }^{4}$ Das Phänomen der ,Krise‘ lässt die Bevölkerung mit einem mulmigen Gefühl zurück, gegen das nur Ablenkung durch andere Themen hilft. Die Notwendigkeit langfristig strukturelle Ursachen der weltweit um sich greifenden Krisen zu bearbeiten, gerät schnell in Vergessenheit, obwohl ihre realen Auswirkungen durchaus ein verheerendes Ausmaß angenommen haben: Ein massives Einbrechen der Mittelschicht in den USA, eine weitreichende Arbeitslosigkeit sowie das Anwachsen der sozialen Problematik in den südeuropäischen Ländern und gravierende wirtschaftliche Nachteile für die sogenannten „Entwicklungsländer“ sind nur einige der Krisenfolgen.

Auf dem Titelblatt des Süddeutsche Zeitung Magazins wird im März 2014 folgende Illustration des spanischen Comicautors Paco Roca veröffentlicht (s. Abb. 1). Unter der Erde versteckt sich ein gigantisches Monster, das im Stil eines Börsenmaklers gekleidet ist. Sein hämisches Grinsen verrät, dass Schlimmes bevorsteht. Unter seinen Krallen befinden sich zahlreiche entwurzelte Wohnhäuser, zu denen die geordnet stehenden Gebäude der Finanzwelt auf der Oberfläche einen deutlichen Kontrast bilden. Die Personifikation der ,Krise‘ durch das Monster erzeugt den Eindruck von Kontrollverlust gegenüber einer zerstörerischen und dennoch bewusst handelnden Machtinstanz. Die Illustration vermittelt die Botschaft, dass die weitaus verheerendere Krise in der Immobilen- und Finanzwirtschaft erst noch bevorsteht.

Ob auf den Finanzmärkten eine neue Krise droht, soll der Beurteilung anderer Fachwissenschaften vorbehalten bleiben. In dieser Arbeit soll hingegen nicht die Tatsächlichkeit von Krisen beleuchtet werden, ihr Ziel ist wichtige Erkenntnisse darüber zu gewinnen, was das Phänomen der ,Krise“ grundlegend charakterisiert. Obwohl es sich bei der ,Krise‘ um einen inflationär auf die verschiedensten Zusammenhänge angewendeten Begriff handelt, gibt es bislang doch nur wenig Erkenntnisse dazu, was das Phänomen genau auszeichnet. Gerade die Rhetorik, als Erforschung strategischer Kommunikationsprozesse, kann einen entscheidenden Beitrag leisten, das diskursive Geschehen rund um die Krisen unserer Zeit besser zu verstehen. So wird sich diese Arbeit nicht das vermeintliche Krisenmonster selbst vorknöpfen, sondern genauer betrachten, was auf der Oberfläche vor sich geht (s. Abb. 2).

4 Vgl. Sinn, H-W. 2016. Der Schwarze Juni: Brexit, Flüchtlingswelle, Euro-Desaster - Wie die Neugründung Europas gelingt. Freiburg: Herder. 


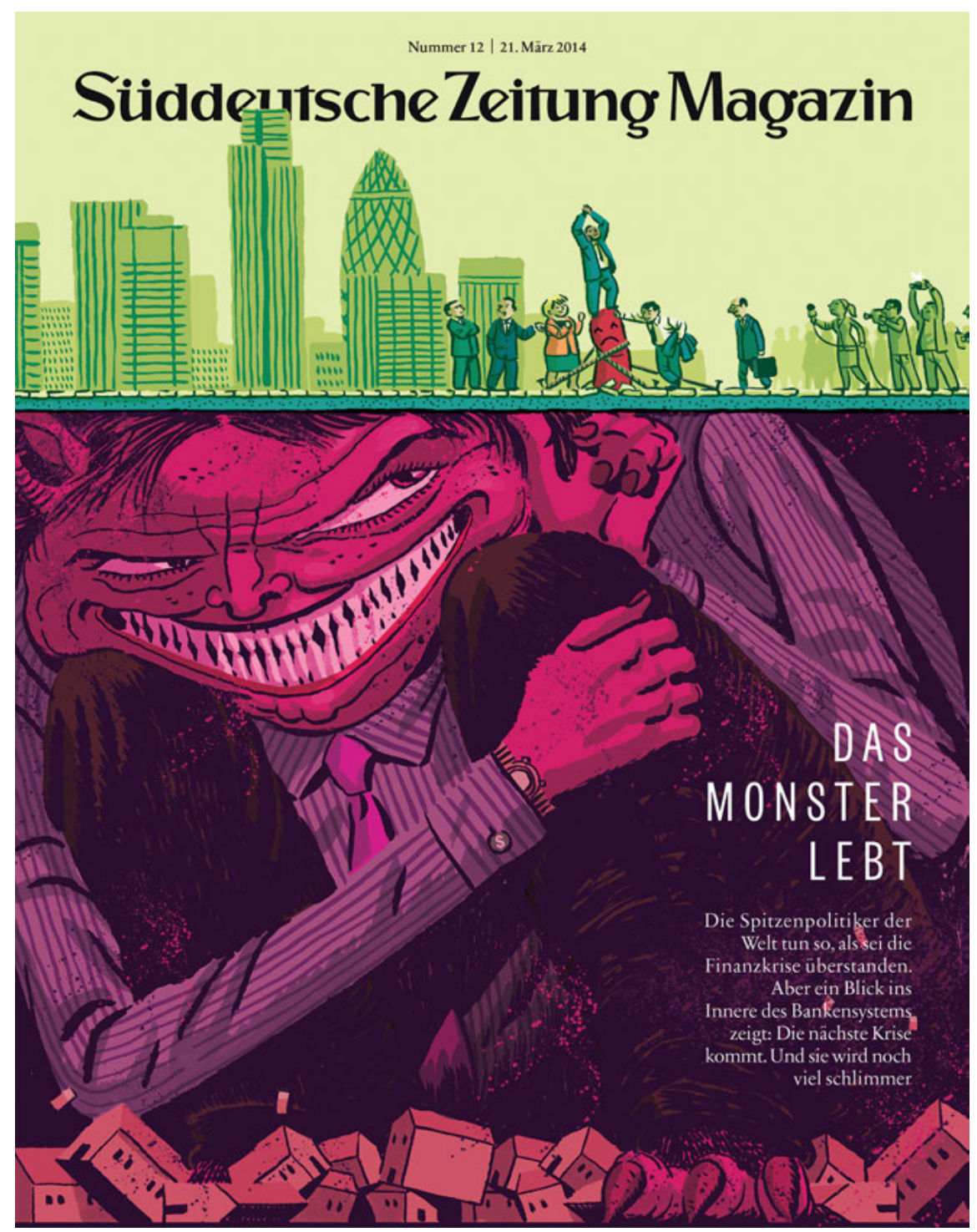

Abb. 1: Titelillustration: Paco Roca. Süddeutsche Zeitung Magazin, Nr. 12, 21. März 2014

Auf dem folgenden Bildausschnitt der Illustration lässt sich ein interessantes Geschehen beobachten. Führende PolitikerInnen feiern ihren Erfolg im Kampf gegen die Krise. Die Dame links neben dem Finger des Monsters, möglicherweise eine Darstellung der Bundeskanzlerin Merkel, reckt die Fäuste. Ein Politiker, der 


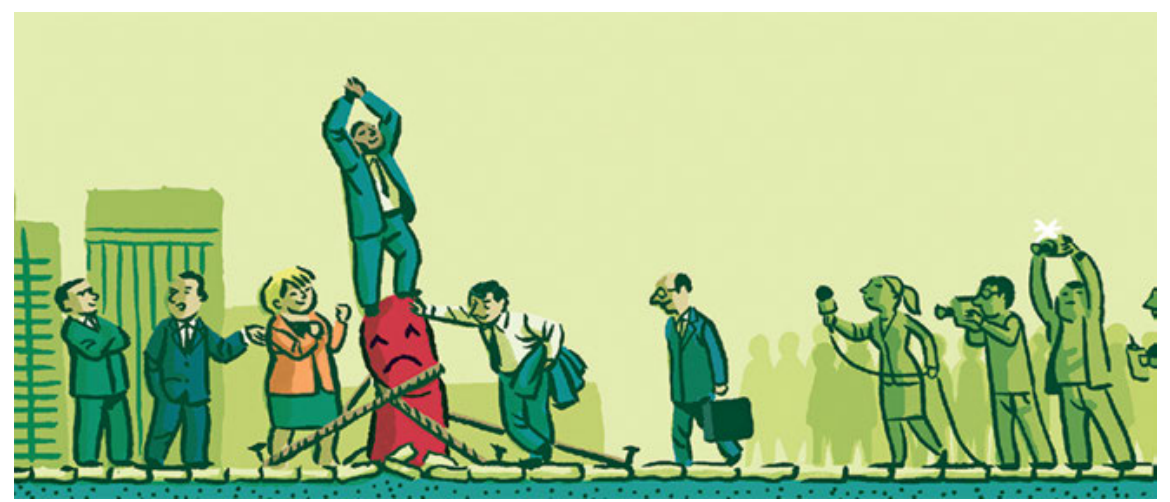

Abb. 2: Ausschnitt der Titelillustration: Paco Roca. Süddeutsche Zeitung Magazin, Nr. 12, 21. März 2014

den Präsidenten Obama darstellen könnte, hat die Spitze des Fingers erklommen und hebt die Hände zur Siegerpose. All dies wird mit Begeisterung von den MedienvertreterInnen dokumentiert und der Öffentlichkeit übermittelt. Diese Darstellung trifft den Kern dieser Arbeit: Wie kann das Phänomen der ,Krise‘ aus rhetorischer Sicht verstanden werden? Zunächst sollen verschiedene Ansätze durchgespielt werden, wie die Krise im rhetorischen System verortet werden kann. Eine mögliche Ursache für den inflationären Gebrauch des Krisenbegriffs ist sein strategischer Nutzen. Als Garant für Aufmerksamkeit lässt er sich vielseitig einsetzen. In dieser Arbeit soll überprüft werden, ob die Krise ein persuasives Mittel darstellt. Das heißt, es wird analytisch herausgearbeitet, wie die Krise sich im politischen Diskurs als Überzeugungsmittel für die eigenen strategischen Ziele nutzen lässt. Dafür werden die Wahlprogramme aller im deutschen Bundestag zwischen 1949 und 2013 vertretenen Parteien herangezogen. Indem unterschiedliche ideologische Perspektiven auf das gesellschaftliche Geschehen beachtet werden, soll ein differenziertes Verständnis für die Überzeugungskraft der Krise geschaffen werden. In der Illustration von Roca scheinen die PolitikerInnen den gefesselten Finger - als Symbol der Krise - zu instrumentalisieren, um ihre eigenen Verdienste und Erfolge der Öffentlichkeit und ihren WählerInnen zu präsentieren. Eine Krise eignet sich demnach zum Eigenlob und liefert gute Gründe für die Wiederwahl der amtierenden SpitzenpolitikerInnen.

Es soll in dieser Arbeit sehr differenziert aufgedeckt werden, ob und wie die Krise von den Parteien der Bundesrepublik als kommunikatives Wirkungsmittel eingesetzt wird, um so ein besseres Verständnis vorherrschender politischer Diskurse zu erhalten. Die Parteien werden im demokratischen System als Repräsentanten unterschiedlicher gesellschaftlicher Ideologien betrachtet. Daraus 
resultiert möglicherweise auch eine abweichende Wahrnehmung der bestimmenden Krisen durch die Parteien. Es wurde ein so langer analytischer Zeitraum gewählt, um ganz unabhängig von bereits diskursiv festgesetzten Krisenzeiträumen ermitteln zu können, welche Zusammenhänge von den Parteien in der BRD als Krisen bezeichnet werden. Bewusst wurde dabei ein Material gewählt, das verschiedene ideologisch motivierte Sichtweisen auf das nationale und internationale Geschehen zeigt. Gerade die durchgängige Beachtung dieser unterschiedlichen Wirklichkeitsperspektiven macht das Alleinstellungsmerkmal dieser Arbeit aus. Es wird kein vermatschter Brei aus Medienberichten durch die Korpusanalyse gedreht, sondern es wird genau zugeordnet, wann welche Partei von einer Krise spricht. Durch eine Topos-Analyse soll ermittelt werden, wie die Parteien die Krise argumentativ einbinden und für welche kommunikativen Ziele sie eingesetzt wird. Anhand des umfassenden Materials von 1949 bis 2013 kann ein übersichtliches Bild erstellt werden, was die Parteien als die Krisen der Nachkriegsgeschichte der BRD bis heute auffassen. Gerade indem zugrundeliegende Strategien hinter der Verwendung des Krisenbegriffs aufgedeckt werden, wird ein besseres Verständnis aktueller Diskurse ermöglicht. 
